\title{
Perfil da Formação Médica em Terapia Intensiva no Estado de Rondônia
}

\author{
The Profile of Medical Education on Intensive \\ Care in the State of Rondônia, Brazil
}

\author{
Luiz Carlos Ufei Hassegawal \\ Ana Lúcia Escobar II \\ Lucia Rejane Gomes da SilvaII \\ Tony Hiroshi Katsuragawa III
}

\author{
PALAVRAS-CHAVE \\ - Cuidados Intensivos. \\ - Unidade de Terapia Intensiva. \\ - Prática Profissional. \\ - Educação Médica.
}

Recebido em: 23/08/2016

Aprovado em: 05/09/2016

\section{RESUMO}

A Medicina Intensiva (MI) é uma especialidade que apresenta um déficit de profissionais qualificados com título em MI. Esse déficit se deve principalmente ao significativo aumento de leitos de unidade de terapia intensiva (UTI) nas últimas décadas. No Brasil, menos de $2 \%$ dos médicos possuem formação nessa especialidade. A legislação vigente preconiza um médico diarista/rotineiro com título de especialista em MI para cada dez leitos em cada turno. O Estado de Rondônia conta com 183 leitos de UTI e apenas 18 médicos intensivistas, segundo a Associação de Medicina Intensiva Brasileira. Esse quadro coloca em risco a qualidade do serviço em MI, principalmente para o usuário. O presente estudo buscou caracterizar o perfil de formação dos médicos intensivistas que atuam nas UTI dos hospitais da rede pública e privada do Estado de Rondônia. Trata-se de um estudo transversal e quantitativo, com uso de questionário autoaplicável com perguntas sobre condições sociodemográficas e perfil profissional, do qual participaram 93 profissionais. Os resultados mostram que a maior proporção é do sexo masculino, na faixa etária de 30 a 40 anos, com renda mensal acima de dez salários mínimos. Cerca de um terço relatou acúmulo de dois locais de trabalho, e menos de $20 \%$ atuam apenas em UTI. A maioria dos participantes atua em UTI pública e privada, e apenas 19,4\% possuem especialização em Medicina Intensiva. A participação em eventos científicos foi significativa, mas apenas um em cada cinco participa de projeto de pesquisa, e um em cada quatro publica artigos científicos. O reduzido número de especialistas que atua nas UTI compromete a qualidade dos serviços de assistência prestados aos usuários. Esforços para correção dessa deficiência devem ser estimulados por meio do aumento da oferta de vagas do programa de residência médica local, visando à melhoria na qualidade da gestão e dos profissionais médicos que atuam nessas UTI.

\begin{abstract}
As a specialization, Intensive Care (IC) is currently experiencing a shortage of qualified professionals with the specific IC title. This shortage is mainly due to a significant increase in the number of beds in intensive care units (ICU) in recent decades, with less than $2 \%$ of physicians trained in this specialization in Brazil. The country's legislation calls for one daytime/routine doctor specialized in IC for every 10 beds per shift, whereas, according to the Brazilian Intensive Care Association, the State of Rondônia only has 183 ICU beds and 18 intensive care physicians. This framework puts the quality of the IC service at risk, particularly for users. The following study sought to characterize the educational profile of intensive care physicians working in intensive care units (ICU) in public and private hospitals in state of Rondônia. Completed by 93 professionals, this transversal and quantitative study featured a self-administered questionnaire containing questions on sociodemographic conditions and professional profiles. The results showed that the highest proportion were male and aged 30-40, with a monthly income over ten minimum monthly salaries. Approximately 1/3 reported working in two different places, and less than $20 \%$ worked exclusively in ICU. Most of the participants worked in public and private ICU, and only $19.4 \%$ were specialized in Intensive Care Medicine. Participation in scientific events was significant, but only 1 out of 5 took part in research projects, with only 1 out of 4 publishing scientific articles. The small number of experts working in ICU compromises the quality of care services provided to users. Efforts to correct this deficiency should be encouraged by increasing the vacancies offered on local residency programs, improving the quality of management, as well as providing better training for medical professionals working in ICU.
\end{abstract}

REVISTA BRASILEIRA DE EDUCAÇÃO MÉDICA 


\section{INTRODUÇÃO}

A partir da década de 1970 e nas três décadas seguintes, houve um expressivo aumento no número de municípios na Amazônia brasileira, principalmente ao longo das rodovias federais, e com isso a oferta de novas oportunidades de trabalho para médicos de todas as especialidades. Com o aumento do sistema sanitário municipal, houve necessidade de interiorização do profissional médico, antes restrito aos grandes centros urbanos ${ }^{1}$. Com a crescente abertura de novas Unidades de Terapia Intensiva (UTI), houve um aumento significativo dos postos de trabalho para médicos prestadores de serviço em UTI, e essa atividade não é exclusividade ou privilégio dos especialistas.

A UTI é definida como ambiente intra-hospitalar de alta tecnologia, composto por equipamentos capazes de monitorizar continuamente, de forma invasiva ou não, pacientes graves ou críticos, descompensados de um ou mais sistemas orgânicos, que, mediante assistência permanente e contínua, são passiveis de recuperação ${ }^{2-5}$. Para isto, necessita-se de médicos altamente qualificados em Medicina Intensiva (MI). Por norma governamental ${ }^{6}$, para a abertura e coordenação de uma UTI é necessário um médico com título de especialista em MI pela Associação de Medicina Intensiva Brasileira (Amib). Atualmente, o principal problema para a assistência médica intensivista em nosso país é a contratação de médicos sem especialização para atuar na grande maioria das UTI, em virtude da grande demanda destes profissionais causada pelo aumento expressivo de novas $\mathrm{UTI}^{7}$.

Um profundo estudo realizado no Brasil, lançado no início de 2013, aponta que em 2020 o número de médicos chegará a $500 \mathrm{mil}^{8}$. Segundo esse estudo, 46,43\% dos médicos em atividade não possuem título de especialista, os denominados generalistas, e Rondônia conta com $56,72 \%$ de médicos generalistas. $\mathrm{Na}$ especialidade de $\mathrm{MI}$, menos de $2 \%$ dos médicos possuem especialização em todo o Brasil, e muitas UTI não têm médicos com essa titulação em seus quadros de trabalho ${ }^{8}$, plantonistas e/ou diaristas.

Este cenário, que não é exclusividade do Brasil ${ }^{9}$, ainda esbarra na dificuldade de contratar o coordenador da UTI, assim como médicos, enfermeiros e fisioterapeutas com mínima experiência para cuidar e tratar dos pacientes graves na $\mathrm{UTI}^{10}$.

A escassez de especialistas em MI também ocorre em países desenvolvidos. Acrescenta-se a este cenário o fato de que os especialistas em MI exercem suas atividades em tempo parcial. Se a atividade fosse dedicada exclusivamente à MI, a prática e a liderança poderiam atingir níveis de elevada qualidade ${ }^{11}$.

De acordo com a Amib, em Rondônia atuam 18 médicos com título de especialista em MI em 16 UTI distribuídas pelo estado $^{12}$. Segundo a Resolução da Diretoria Colegiada $n^{0} 7$ (RDC nº 7 de 24/02/2010), da Agência Nacional de Vigilância Sanitária ${ }^{6}$, é necessário um médico intensivista para cada dez leitos de UTI. Em Rondônia, há um total de 183 leitos para pacientes críticos $^{13}$, e somente na capital Porto Velho estão em funcionamento 12 UTI que totalizam 143 leitos.

Considerando este cenário, o presente estudo teve como alvo a caracterização do perfil de formação e socioeconômico de médicos que atuam nas UTI dos hospitais de gestão pública e privada do Estado de Rondônia, Amazônia Ocidental, Brasil.

\section{METODOLOGIA}

Trata-se de um estudo transversal sobre o perfil de formação dos médicos atuantes nas UTI adulto do Estado de Rondônia. Os dados da pesquisa foram coletados de setembro a dezembro de 2014.

Rondônia é composta por 52 municípios, com população total estimada em cerca de 1,748 milhão de habitantes no ano de $2014^{14}$. A distribuição entre os sexos apresenta discreto predomínio masculino ${ }^{13}$. A coleta de dados foi realizada em todas as UTI do estado, localizadas nas cidades de Porto Velho, Ariquemes, Ji-Paraná, Cacoal e Vilhena, de gestão pública e privada.

Os dados foram coletados por meio de questionário estruturado, adaptado e autoaplicável ${ }^{15,16}$, mesclando perguntas de resposta aberta e fechada. O questionário foi aplicado em entrevista pessoal a cada participante e após concordância e assinatura do Termo de Consentimento Livre e Esclarecido (TCLE).

O questionário abordou variáveis sociodemográficas, como sexo, idade, estado civil, escolaridade, tempo de trabalho, tempo de trabalho na instituição, jornada de trabalho, carga horária diária e semanal, remuneração salarial, educação continuada. Foram incluídas, no componente específico do questionário, perguntas voltadas para verificar a qualificação do médico no atendimento às UTI. Estas questões versaram sobre formação médica, qualificação, atualização e tempo de atuação na área.

Os dados sociodemográficos foram submetidos a análise estatística descritiva por meio das frequências absolutas e relativas, utilizando-se os programas Microsoft Office ExcelÒ e SPSSÒ (Statistical Package for the Social Sciences IBM for Windows, Version 20.0. Armonk, NY: IBM Corp.).

Os resultados foram expressos em números e percentuais. A análise sobre especialistas e tempo de graduação foi comparada por meio do teste qui-quadrado, com nível de significância de $5 \%$. O tempo de graduação foi considerado igual ou inferior a 
cinco anos, e superior a cinco anos, devido ao tempo necessário à realização da residência em UTI, que é de quatro anos no Brasil ${ }^{17}$.

A pesquisa se fundamentou nos preceitos éticos da Resolução 466, de 12 de dezembro de 2012, do Conselho Nacional de Saúde, que trata das Diretrizes e Normas de Pesquisa Envolvendo Seres Humanos ${ }^{18}$. O estudo foi aprovado pelo Comitê de Ética em Pesquisa (CEP) do Núcleo de Saúde da Universidade Federal de Rondônia, sob o número CAAE: 34863814.1.0000.5300.

\section{RESULTADOS}

A população do estudo foi composta por todos os médicos que estavam efetivamente lotados nas UTI $(n=122)$ e que assinaram o TCLE ( $\mathrm{n}=93$ ). Foram excluídos aqueles que estavam em período de férias ou licença trabalhista no momento da coleta de dados. O total de participantes foi de 93 médicos que atuam em UTI no Estado de Rondônia.

Entre os participantes, 78,5\% (73/93) eram homens, e 28\% (26/93) apresentaram tempo de graduação $\leq 5$ anos (Figura 1). A média geral da idade foi de 39,3 anos, sendo que para o sexo feminino foi de 34,5 anos (mínimo: 26; máximo: 51), e 40,7 anos (mínimo: 26; máximo: 64) para o sexo masculino.

Figura 1

Distribuição da idade da população do

estudo, por gênero e tempo de graduação

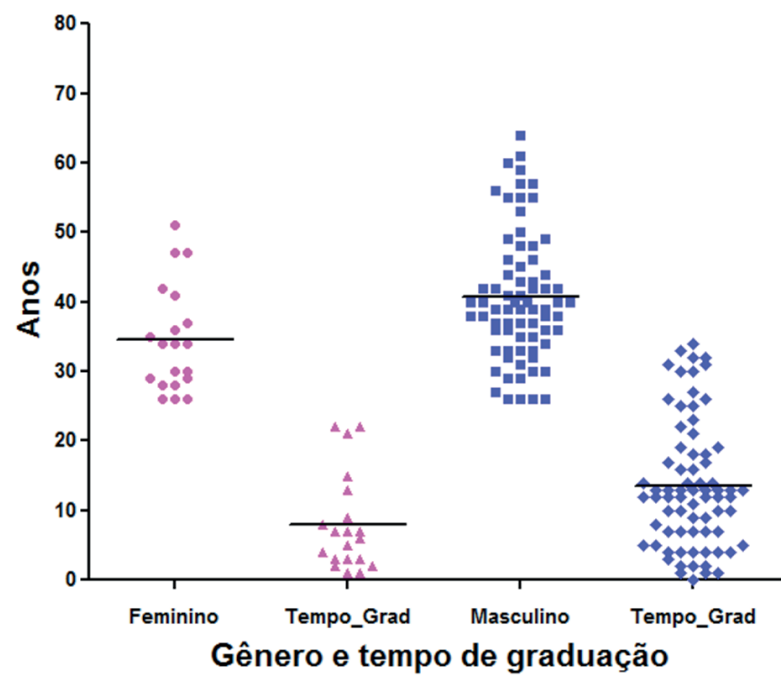

Nota: Tempo Grad = tempo de graduação

Do total, 40,9\% (38/93) tinham entre 30 e 40 anos de idade, e 94,6\% (88/93) declararam possuir renda mensal acima de $\mathrm{R} \$ 8.000,00$. Os que trabalhavam exclusivamente na es- pecialidade de MI representaram somente 18,3\% (17/93), e $81,7 \%$ (76/93) atuavam em mais de uma UTI, sendo que $68,8 \%$ (64/93) exerciam suas atividades nos setores público e privado (Tabela 1).

TABELA 1

Características socioeconômicas dos médicos que atuavam em Medicina Intensiva adulto no Estado de Rondônia em 2014

\begin{tabular}{|c|c|c|}
\hline Variáveis & $\mathbf{N}$ & $\%$ \\
\hline \multicolumn{3}{|l|}{ Faixa etária } \\
\hline$<30$ & 14 & 15,0 \\
\hline $30-35$ & 16 & 17,2 \\
\hline $35-40$ & 22 & 23,7 \\
\hline $40-50$ & 28 & 30,1 \\
\hline $50-60$ & 10 & 10,8 \\
\hline${ }^{3} 60$ & 3 & 3,2 \\
\hline \multicolumn{3}{|l|}{ Renda mensal em $\mathrm{R} \$ *$} \\
\hline$<3.000$ & 1 & 1,1 \\
\hline $3.000-5.000$ & 1 & 1,1 \\
\hline $5.000-8.000$ & 3 & 3,2 \\
\hline${ }^{3} 8.000$ & 88 & 94,6 \\
\hline \multicolumn{3}{|c|}{ Quantidade de locais de trabalho (Medicina Intensiva) } \\
\hline 1 & 17 & 18,2 \\
\hline 2 & 33 & 35,5 \\
\hline 3 & 29 & 31,1 \\
\hline 4 & 12 & 12,9 \\
\hline 5 & 2 & 2,2 \\
\hline \multicolumn{3}{|c|}{ Trabalho exclusivo em Unidade de Terapia Intensiva } \\
\hline Sim & 17 & 18,3 \\
\hline Não & 76 & 81,7 \\
\hline \multicolumn{3}{|c|}{ Tempo de atuação em Medicina Intensiva (em anos) } \\
\hline$<5$ & 39 & 41,9 \\
\hline $5-10$ & 30 & 32,2 \\
\hline $10-15$ & 8 & 8,6 \\
\hline $15-20$ & 9 & 9,7 \\
\hline $20-25$ & 2 & 2,2 \\
\hline $25-30$ & 3 & 3,2 \\
\hline${ }^{3} 30$ & 2 & 2,2 \\
\hline \multicolumn{3}{|c|}{ Local de trabalho (gestão da Unidade) } \\
\hline Exclusivamente pública & 18 & 19,4 \\
\hline Exclusivamente privada & 11 & 11,8 \\
\hline Púbica e privada & 64 & 68,8 \\
\hline
\end{tabular}

Nota: *Salário mínimo de referência: $R \$ 724,00$.

Em relação ao perfil profissional (Tabela 2), somente 18 $(19,4 \%)$ eram especialistas em MI através de residência ou de certificação pela Amib em convênio com a Associação Médica Brasileira (AMB), e, destes, somente 4,3\% (4/93) fizeram residência médica em MI. Quanto à atualização profissional, observa-se que 90,3\% (84/93) participaram de até dez eventos nos últimos cinco anos. 


\begin{tabular}{|c|c|c|}
\hline \multicolumn{3}{|c|}{$\begin{array}{c}\text { TABELA } 2 \\
\text { Perfil profissional e de atualização de } \\
\text { médicos que atuavam em Medicina Intensiva } \\
\text { no Estado de Rondônia em } 2014\end{array}$} \\
\hline Variáveis & $\mathbf{N}$ & $\%$ \\
\hline \multicolumn{3}{|c|}{ Especialização em Medicina Intensiva (residência ou título) } \\
\hline Sim & 18 & 19,4 \\
\hline Não & 75 & 80,6 \\
\hline \multicolumn{3}{|c|}{$\begin{array}{l}\text { Participação em eventos científicos nos últimos cinco anos } \\
\text { (números absolutos) }\end{array}$} \\
\hline$\leq 10$ & 84 & 90,3 \\
\hline$>10$ & 9 & 9,7 \\
\hline \multicolumn{3}{|c|}{ Assinatura de periódicos de qualquer área } \\
\hline Sim & 65 & 69,9 \\
\hline Não & 28 & 30,1 \\
\hline \multicolumn{3}{|c|}{ Participação em projeto de pesquisa } \\
\hline Sim & 15 & 16,1 \\
\hline Não & 78 & 83,9 \\
\hline \multicolumn{3}{|c|}{ Publicação de artigos em qualquer área } \\
\hline Sim & 24 & 25,8 \\
\hline Não & 69 & 74,2 \\
\hline
\end{tabular}

Dos participantes, 16,1\% (15/93) faziam parte de algum projeto de pesquisa (Tabela 2). Proporcionalmente, 33,3\% (6/18) dos médicos especialistas participaram de algum projeto de pesquisa, ao passo que, entre os não especialistas, apenas $12,0 \%$ (9/75) o fizeram. Contudo, não há diferença estatisticamente significativa entre ter ou não a especialização em MI e a participação em projeto de pesquisa $(\mathrm{p}=0,07)$. Com referência à produção científica, apenas 25,8\% (24/93) dos participantes publicaram algum artigo científico, e a proporção de especialistas e não especialistas foi de 33,3\% (6/18) e 24,0\% (18/75), respectivamente $(\mathrm{p}=0,54)$.

Para as variáveis de tempo de graduação e especialista em MI, observa-se que todos os intensivistas com título e/ou residência médica em MI $(18 / 93 ; 19,4 \%)$ tinham mais de cinco anos de graduação (Figura 2), sendo este achado estatisticamente significante $(\mathrm{p}<0,005)$.

\section{DISCUSSÃO}

Há um total de 4.257 leitos hospitalares no Estado de Rondônia ${ }^{13}$, o que corresponderia à necessidade de no mínimo 255 leitos de UTI (6\%). Portanto, há uma defasagem de 72 leitos para pacientes críticos ${ }^{19}$ e de pelo menos sete especialistas em MI somente para a gestão de tais unidades.
Figura 2

Número de médicos com formação em Medicina Intensiva, residência ou especialização

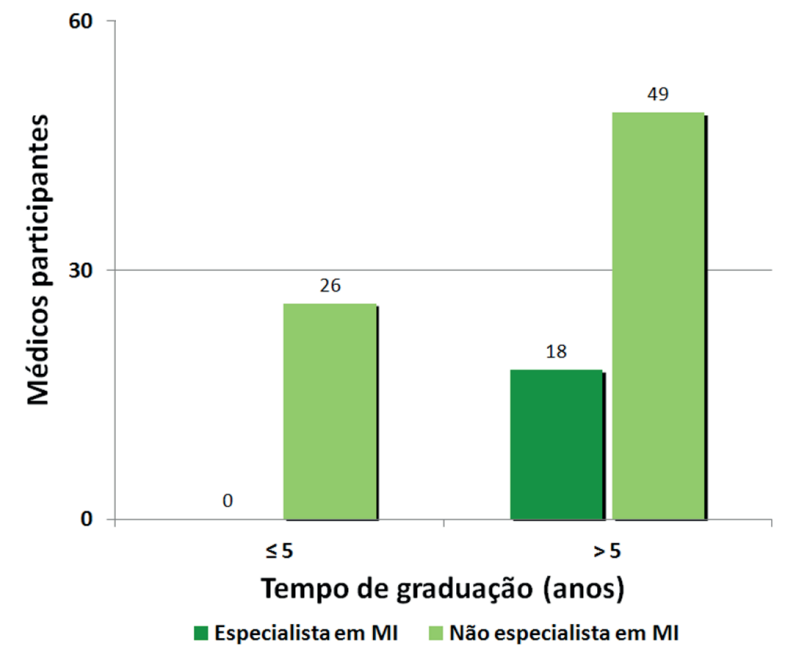

Nota: $M I=$ Medicina Intensiva. A diferença entre o tempo de formação foi significativa $(p=0,001)$

A quantidade de médicos que trabalham em UTI adulto sem formação específica em Rondônia é elevada, alcançando $75 \%$. Inicialmente, acreditou-se que esses valores estariam relacionados ao pequeno número de vagas ofertadas de residência médica em MI, o que levaria à formação de profissionais em número insuficiente para suprir a necessidade das novas UTI. Entretanto, segundo dados da Comissão de Residência Médica do Hospital de Base Dr. Ary Pinheiro (Coreme-HB), $50 \%$ das quatro vagas de residência de MI oferecidas em 2014 e 2015 ficaram ociosas, revelando que esta não é a razão da ausência de médicos com formação no estado ${ }^{20,21}$.

Quanto aos postos de trabalho, houve um incremento de $34 \%$ nos leitos de UTI entre os anos de 2012 e 2014, tendo como reflexo o seu preenchimento com médicos sem qualificação para a área de atuação ${ }^{22}$.

A falta de especialistas em MI e a permissividade da legislação vigente ${ }^{6}$ levam as instituições de saúde a contratar médicos não especialistas, que têm a oportunidade de trabalho para depois realizar uma residência médica, não especificamente em $\mathrm{MI}^{23}$.

A expressiva falta de especialistas encontrada deve-se também à ausência de normatização quanto à admissão de profissionais para atuarem em MI. A RDC 07 não especifica a formação mínima do profissional médico para atuar em UTI, principalmente em regime de plantão ${ }^{6}$. A escassez de médicos intensivistas especializados não é exclusividade das UTI de 
Rondônia. Em países desenvolvidos, como os Estados Unidos, a carência desta força de trabalho persiste há mais de uma década $^{11}$. Entretanto, a presença do médico especializado e capacitado é fundamental para a melhoria dos serviços necessários numa $\mathrm{UTI}^{24}$.

A presença nas UTI de médicos com tempo de graduação inferior a cinco anos demonstra a falta de especialização em MI desses profissionais. Há uma diferença estatisticamente significativa entre o número de médicos com especialização em MI com o tempo de graduação maior que cinco anos ( $p$ $<0,005)$. A média nacional de médicos especialistas em MI é de $46,9 \operatorname{anos}^{8}$, e a proporção por sexo mostrou predominância do sexo masculino na proporção de 7:3. No presente estudo, a média de idade foi de 40,8 anos, e o predomínio masculino foi de 5:1. Isto sugere que os especialistas em MI do presente estudo são ligeiramente mais jovens e com predomínio do sexo masculino superior à média nacional.

Em 2005, um estudo que abrangeu a Região Norte já enfatizava que a maioria dos médicos necessitava aprimorar seus conhecimentos e melhorar suas qualificações técnicas ${ }^{25}$. Os resultados apresentados no presente estudo sugerem que os médicos que atuam nas UTI de Rondônia buscam melhorar seus conhecimentos. Contudo, a produção científica gerada ainda é muito reduzida, condizente com outros estudos ${ }^{15,16}$. Com os avanços tecnológicos e a dinâmica dos protocolos utilizados na MI, a qualificação dos profissionais médicos é de extrema importância para o atendimento ao paciente crítico.

\section{CONCLUSÃO}

Os resultados apontam baixo interesse dos médicos em se especializar em MI e também a reduzida proporção de especialistas em nossas UTI. A maioria dos médicos que atuam nas UTI adulto no Estado de Rondônia não possui especialização em MI, mostrando que merecem atenção especial de seus gestores, das autoridades reguladoras e da sociedade organizada.

Isso compromete a qualidade dos serviços de assistência prestados aos usuários e, consequentemente, poderia aumentar a morbimortalidade no setor. Esforços estimulantes devem ser concentrados para corrigir essas deficiências, que não dependem de recursos financeiros e se refletem diretamente na melhoria da qualidade na gestão dos serviços de saúde.

Os autores do presente estudo propõem que o programa de residência médica local em MI seja incentivado a incrementar o número de vagas ofertadas, alinhando-se às carências observadas e com o objetivo de suprir a demanda de especialistas em MI a médio e longo prazo.

\section{REFERÊNCIAS}

1. Campos FE, Machado MH, Girardi SN. A fixação de profissionais de saúde em regiões de necessidades. Divulg Saúde Debate 2009;44:13-24.

2. Tranquitelli AM, Ciampione MHT. Número de horas de cuidados de enfermagem em unidade de terapia intensiva de adultos. Rev Esc Enf USP 2007;41(3):371-77.

3. Azoulay E, Mancebo J, Brochard L. Surviving the night in the ICU: who needs senior intensivists? Am J RespirCrit Care Med 2010;182(3):293-4.

4. Estenssoro E, Barbas CS, Román LS. ICU staffing: the South American perspective. Am J RespirCrit Care Med 2010;182(4):441-2

5. Cremesp. Conselho de Medicina do Estado de São Paulo. Resolução CREMESP nº 71, de 08 de novembro de 1995. [capturado em 24 mai 2014]. Disponível em: <http:// www.medici naintensiva.com.br/cremesp.htm $>$.

6. Anvisa. Agência Nacional de Vigilância Sanitária. Resolução - RDC nº 7, de 24 de fevereiro de 2010. Dispõe sobre os requisitos mínimos para funcionamento de Unidades de Terapia Intensiva e dá outras providências. Diário Oficial da União25 fev 2010; Seção 1.

7. Kelley MA, Angus D, Chalfin DB, Crandall ED, Ingbar D, Johanson W, et al. The Critical Care Crisis in the United States: A Report from the Profession. Chest. 2004;125:1514-17.

8. Scheffer M (coord.), Cassenote AJF, Biancarelli A. Demografia médica no Brasil. CFM e CREMESP 2013. 256p.

9. Piquette D, Fowler RA, Slutsky AS. Impact of intensivist staffing on patient care and trainee education: a Canadian perspective. Am J RespirCritCareMed 2010;182(4):442-4.

10. Tironi MOS, Sobrinho CLN, Barros DS, Reis EJFB, Marques Filho ES, et al. Trabalho e síndrome da estafa profissional (Síndrome de Burnout) em médicos intensivistas de Salvador. Rev Assoc Med Bras 2009;55(6):656-62.

11. Pastores SM, Kevtan V. Falta de Especialistas em terapia intensiva nos Estados Unidos: percepções recentes e soluções propostas. Rev Bras Ter Intensiva 2015;27(1):5-6.

12. Amib. Associação Brasileira de Medicina Intensiva. Disponível em: <http:/ / www.amib. org.br/>. Acesso restrito Associado.

13. Anvisa. Agência Nacional de Vigilância Sanitária. Cadastro Nacional de Estabelecimento de Saúde (CNESnet). [capturado em 01 abr. 2015]. Disponível em: <http:/ / cnes. datasus.gov.br/Lista_Tot_Es_Municipio.asp?Estado=11\& NomeEstado=RONDÔNIA>

14. Brasil. Instituto Brasileiro de Geografia e Estatística. Projeção da população 2000-2030. [capturado em 18 abr, 2015]. 
Disponível em:<http://www.ibge.gov.br/estadosat/perfil.php? sigla $=$ ro $>$.

15. Lacerda JCD. Perfil profissional do intensivista pediátrico no Estado do Rio de Janeiro. Rio de Janeiro; 2011. Mestrado [Dissertação] -Universidade Federal do Rio de Janeiro, Centro de Ciências da Saúde, Faculdade de Medicina.

16. Lacerda JCD, Barbosa AP, Cunha AJLA. Perfil profissional do intensivista pediátrico no Estado do Rio de Janeiro, Sudeste do Brasil.Rev Bras Ter Intensiva. 2011; 23(4):462-69.

17. Brasil. Ministério da Educação. Comissão Nacional de Residências Médica. Resolução CNRM nº 02/2006, de 17 de maio de 2006. Dispõe sobre requisitos mínimos dos Programas de Residência Médica e dá outras providências. Diário Oficial da União17 maio 2006, Seção 1.

18. Brasil. Ministério Da Saúde. Conselho Nacional de Saúde. Resolução № 466 de 12 de Dezembro de 2012. Diário Oficial da União13 junho 2013, Seção 1.

19. Amib. Associação Brasileira de Medicina Intensiva. Regulamento técnico para funcionamento de unidades de terapia intensiva. AMIB. Comissão de Defesa do Exercício Profissional. 24 abr. 2009. [capturado em 15 jan. 2015]. Disponível em: <http://www.amib. org.br/fileadmin/RecomendacoesAMIB.pdf $>$.

20. Rondônia. Secretaria de Estado da Saúde de Rondônia. Comissão de Residência Médica do Hospital de Base “Dr. Ary Pinheiro". Edital 001/2015/COREME/HBAP/SESAU. Diário Oficial do Estado de Rondônia № 261812 janeiro 2015.

21. Rondônia. Secretaria de Estado da Saúde de Rondônia. Comissão de Residência Médica do Hospital de Base "Dr. Ary Pinheiro". Edital 002/2015/COREME/HBAP/SESAU. Diário Oficial do Estado de Rondônia № 265913 março 2015.

22. Rondônia. Secretaria de Estado da Saúde de Rondônia. Em dois anos, a oferta de leitos de UTI em Rondônia cresceu $34 \%$. Portal do Governo do Estado de Rondônia. [capturado em 20 ago. 2015]. Disponível em: <http:/ / www.rondonia.ro.gov.br/2015/03/47446/>.
23. Sobrinho CLN, Barros DDS, Tironi MOS, Filho ESM. Médicos de UTI: prevalência da Síndrome de Burnout, características sociodemográficas e condições de trabalho. Rev Bras Educ Méd. 2010; 34(1):106-15.

24. Coutright KR, Kerlin MP. A unidade de terapia intensiva e a qualidade dos cuidado: desafios nos tempos de poucos intensivistas. Rev Bras Ter Intens. 2014; 26(3):205-207.

25. Andrade EO, Gouveia VV, Carneiro MB, Pinheiro AG, (coords). $\mathrm{O}$ médico e seu trabalho: resultados da região Norte e seus Estados. Conselho Federal de Medicina, Brasília. 2005. 285p. Disponível em: <http://www.portalmedico. org.br/include/biblioteca_virtual/medico_e_seu_trabalho/regiao_norte/NORTE.pdf $>$.

\section{CONTRIBUIÇÃO DOS AUTORES}

Concepção: LCUH, ALE, LRGS.

Análise formal: LCUH, ALE, LRGS, THK.

Investigação: LCUH.

Metodologia: LCUH, ALE, LRGS.

Recursos: LCUH.

Validação: LCUH, THK.

Escrita — rascunho original: LCUH, THK

Escrita — revisão e edição: LCUH, THK.

\section{CONFLITO DE INTERESSES}

Os autores declaram não haver conflito de interesses.

\section{ENDEREÇO PARA CORRESPONDÊNCIA}

Tony Hiroshi Katsuragawa

Centro de Pesquisa em Medicina Tropical - Cepem Laboratório de Epidemiologia

Av Guaporé, 215

Lagoa - Porto Velho

CEP 76812-329 - RO

E-mail: tonykatsuragawa@yahoo.com.br 